EPJ Web of Conferences 116, 11006 (2016)

DOI: $10.1051 /$ epjconf/201611611006

(C) Owned by the authors, published by EDP Sciences, 2016

\title{
Results from the ANTARES neutrino telescope
}

\author{
M. Spurio, on behalf of the ANTARES Collaboration ${ }^{1,2, a}$ \\ ${ }^{1}$ Dipartimento di Fisica e Astronomia Università di Bologna, Italy \\ ${ }^{2}$ INFN- Sez. di Bologna, Viale Berti Pichat 6/2, 40127 Bologna, Italy
}

\begin{abstract}
ANTARES is the largest neutrino telescope in the Northern hemisphere, running in its final configuration since 2008. After the discovery of a cosmic neutrino diffuse flux by the IceCube detector, the search for its origin has become a key mission in highenergy astrophysics. The ANTARES sensitivity is large enough to constrain the origin of the IceCube excess from regions extended up to $0.2 \mathrm{sr}$ in the Southern sky. The Southern sky has been studied searching for point-like objects, for extended regions of emission (as the Galactic plane) and for signal from transient objects selected through multimessenger observations. Upper limits are presented assuming different spectral indexes for the energy spectrum of neutrino sources. In addition, ANTARES provides results on studies of the sky in combination with different multimessenger experiments, on atmospheric neutrinos, on the searches for rare particles in the cosmic radiation (such as magnetic monopoles and nuclearites), and on Earth and Sea science. Particularly relevant are the searches for Dark Matter: the limits obtained for the spin-dependent WIMP-nucleon cross section overcome that of existing direct-detection experiments. The recent results, widely discussed in dedicated presentations during the $7^{\text {th }}$ edition of the Very Large Volume Neutrino Telescope Workshop (VLVvT-2015), are highlighted in this paper.
\end{abstract}

\section{Introduction}

The ANTARES underwater neutrino telescope is located $40 \mathrm{~km}$ off the coast of Toulon, France and anchored to the seabed at a depth of $2.5 \mathrm{~km}$. It consists of an array of 885 photomultiplier tubes (PMTs) of 10 inches covering an instrumented volume of approximately $0.01 \mathrm{~km}^{3}$, and it is designed primarily to search for charged-current interactions of neutrinos with $E_{v}>100 \mathrm{GeV}$. Results from different analyses are reported here and in [1]. These include several measurements which are used to constrain both pointlike (Sect. 2) and extended (Sect. 3) origins of the astrophysical flux observed by IceCube [2]; a new reconstruction of showers which allows a point-source search with cascade events (Sect. 4); limits on indirect searches for dark matter (Sect. 5).

\footnotetext{
ae-mail: spurio@bo.infn.it
}

This is an Open Access article distributed under the terms of the Creative Commons Attribution License 4.0, which permits unrestricted use, distribution, and reproduction in any medium, provided the original work is properly cited. 

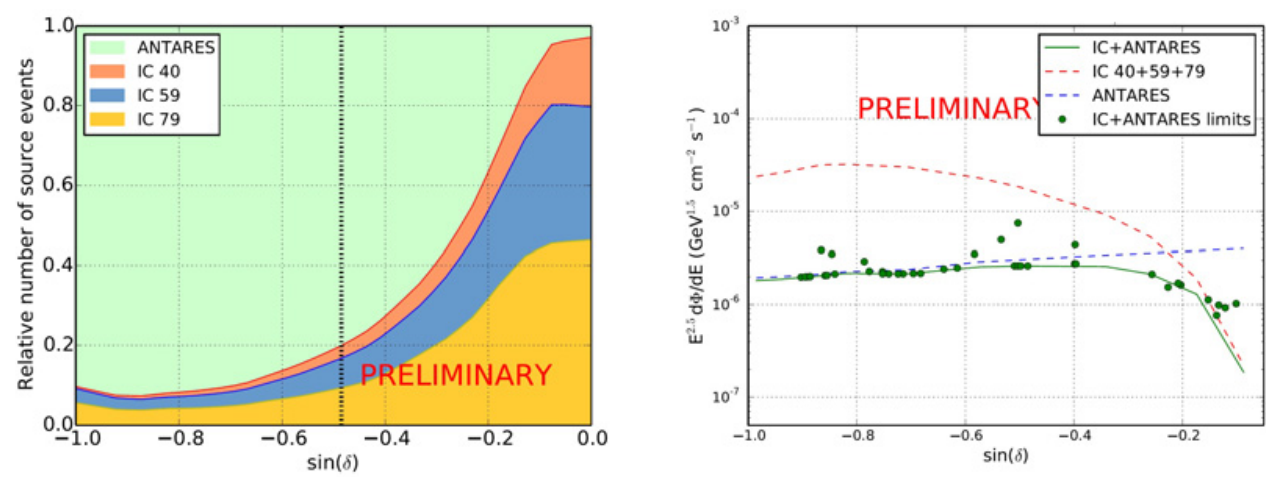

Figure 1. Left: Fractional contributions of each data set to the total number of signal events passing cuts in the joint ANTARES-IceCube analysis, for sources with an $\mathrm{E}^{-2.5}$ spectrum, as a function of sinus of the declination $\delta$. Right: Sensitivities (lines) and limits (dots) to an $\mathrm{E}^{-2.5}$ flux, using ANTARES (blue), IceCube (red), and combined (green) data, as a function of $\sin \delta$.

\section{Searches for astrophysical neutrino point sources}

Astrophysical point-like sources of neutrinos can be individuated looking for an excess of muons from the interactions of $v_{\mu}$ in the proximity of the detector. The high rate of downgoing muons from the interactions of cosmic rays (CRs) in the Earth's atmosphere restricts such searches to events coming from below, or only a few degrees above the horizon. The primary background to such searches is due to atmospheric neutrinos and those few atmospheric muons mis-reconstructed as up-going. The long scattering length of light in seawater provides an excellent directional resolution on the $v_{\mu}$ of $\sim 0.4^{\circ}$ for an $E^{-2}$ source [3]. This allows a strong suppression of both backgrounds in the signal direction, and a correspondingly good sensitivity to neutrino sources located in the Southern Hemisphere.

A joint ANTARES and IceCube search for a neutrino excess from selected sources in the Southern hemisphere is detailed in [4]. The relative number of source events expected to be present in each data set is shown in Fig. 1 (left) for an $E^{-2.5}$ spectrum, the current best-fit to the cosmic neutrino flux from IceCube [2]. The ANTARES contribution is dominant for declination $<-15^{\circ}$. In fact, ANTARES is more sensitive to tracks produced by (relatively) low-energy $v_{\mu}$, while IceCube requires high-energy events to distinguish them from the huge background due to atmospheric muons. The overall sensitivities of the two detectors is also a function of the background rates, and of the angular and energy resolutions, which are not shown. The results of the combined search are shown in Fig. 1 (right), for an $E^{-2.5}$ source spectrum. No significant cluster is found and the combined analysis improves limits set by each experiment.

The cosmic $v$ signal observed by IceCube is due to high-energy events starting in the fiducial volume. Most of these events (shortened as HESE) are showers for which the angular determination is poor (10-20 degrees). No significant fluctuation over a diffuse flux has been observed so far. However, the clustering of some events is intriguing, suggesting the possibility of a Galactic component in the cosmic signal [5]. In [6], it was argued that the HESE cluster near the Galactic plane [7] could be due to a single source, not identified because of the poor angular resolution. ANTARES searched for a possible excess in a wide region near the Galactic center, without any positive result. These limits the flux of such a source as a function of spectral index, shown by the solid lines in Fig. 2.

AGN have long been proposed as a source of high-energy CRs and, hence, neutrinos. Blazars (AGN with jets pointed towards the Earth), exhibit bright flares which dominate the extragalactic $\gamma$-ray sky observed by Fermi-LAT and IACTs. Using multi-wavelength observations, several bright blazars have 


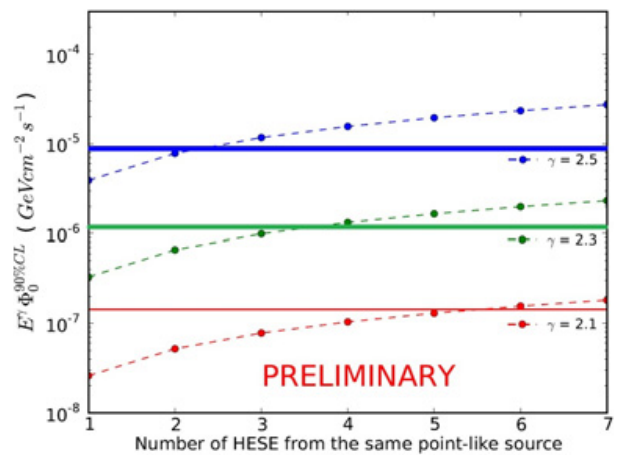

Figure 2. ANTARES limits (solid lines) at 90\% C.L. on the contribution of point-like sources to the IceCube HESE sample for different spectral indices, shown for a source at $\delta=-29^{\circ}$. These are compared to (dashed lines) the flux required to produce a given expected number of HESE, according to the computation in [5]. The range where the latter is greater than the former is excluded. ANTARES can thus rule out any single point-source of neutrinos in the region of the Galactic Centre with spectral index of -2.5 as having a flux corresponding to more than 2 HESE.

been reported by the TANAMI collaboration [8] to lie within the 50\% error bounds of the reconstructed arrival directions of the PeV-scale events IC 14 and IC 20 observed by IceCube. ANTARES observes signal-like events from the two brightest blazars in the field of IC 20 [9], although this is also consistent with background fluctuations. The highest-energy "Big Bird" event (IC 35) was detected during an extremely bright flare from the blazar PKS B1424-418, which lies within the 50\% error region of the IC 35 arrival direction. No ANTARES event was found from this source during the flaring period, constraining severely the model in [8].

Another analysis [10] targets a sample of 41 blazar flares observed by Fermi-LAT and 7 by the IACTs. The lowest pre-trial p-value of 3.3\% was found for the blazar 3C 279, which comes from the coincidence of one event with a 2008 flare. However, the post-trial p-value is not significant. Similar methods were also used to search for neutrino emission during the flares from galactic $\mathrm{x}$-ray binaries. A total of $34 \mathrm{x}$-ray- and $\gamma$-ray-selected binaries were studied, with no significant detections, allowing some of the more optimistic models for hadronic acceleration in these sources to be rejected at $90 \%$ C.L. (paper in preparation). Independent searches for spatial (through two-point correlation method) and temporal collective effects in the ANTARES data have been presented by R. Gracia and A. Coleiro in [1] and by A. Kouchner at this conference.

Under particular conditions, a dedicated program (denoted as TAToO) sends an alert message to trigger robotic optical telescopes, and/or the Swift-XRT. The very short alert-generation time (a few seconds) and half-sky simultaneous coverage of ANTARES makes it ideal for detecting transient signals. Results from 42 optical and $7 \mathrm{x}$-ray alerts occurred from 2011 and the beginning of 2015 are reported in [11]. No associated transient event was detected, and this non-observation was used to place limits on the astrophysical origin of the detected neutrinos. It should be mentioned that on September $1^{\text {st }}, 2015$, the XRT onboard Swift followed an ANTARES high-energy neutrino, detecting an uncatalogued x-ray source at 8 arcmin from the neutrino direction [12]. Different multi-wavelength observations (from IR, optic, radio, $\mathrm{x}$-ray and $\mathrm{TeV}$ devices) have followed the alerts. The $\mathrm{x}$-ray follow-up of this source during five days has shown an x-ray flare of few days large. Optical data have indicated a bright star in the same location of the XRT source without significant variability. Additional multi-wavelength data have permitted to identify it as a young stellar object. There is therefore a very low probability that the X-ray source is associated with the observed neutrino. 


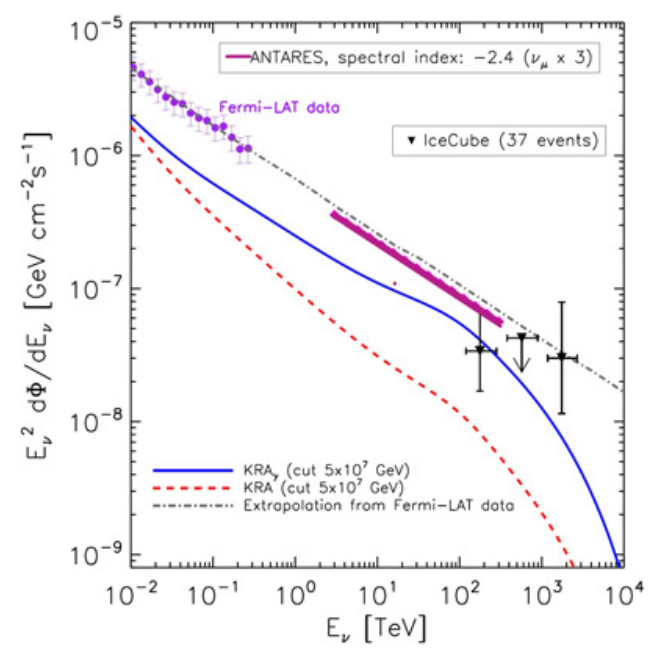

Figure 3. ANTARES upper limit for the flux of neutrinos from the Galactic Center, $|l|<40^{\circ}$ and $|b|<3^{\circ}$ (see L. Fusco in [1]). The result is compared to the expected neutrino flux (dot-dashed line) extrapolated from the Fermi-LAT diffuse flux up to IceCube energies [2] and to the neutrino expectations as computed in [14] in the same Galactic Plane region.

\section{Searches for extended sources in the Southern sky}

Several extended galactic regions (Fermi Bubbles, Galactic Plane, and the IceCube cluster) have been proposed as hadronic acceleration sites. Some models involve CRs that produce pions and, hence, neutrinos from collisions with the interstellar medium during propagation in our galaxy. Some evidence for these processes comes from observations by Fermi-LAT. In particular, the Fermi Bubbles are giant regions of $\gamma$-ray emission extending out of the galactic center, and are proposed as hadronic acceleration sites with neutrinos expected from p-p collisions. ANTARES searches for an excess in the neutrino flux from these regions use "on-zones" defined by specific templates. The number of events from on-zones is compared to that from "off-zones" of exactly the same size and shape, but offset in right ascension. Thus, the off-source regions give an unbiased estimate of the background in the source region in a way that is independent of simulations. The background from the atmospheric neutrinos is suppressed using the energy estimate of the neutrino candidates.

Upper limits from the neutrino emission from the Fermi Bubbles were already set in [13]. The recent update of this analysis (see S. Hallmann in [1]) shows a slight excess in the source region, corresponding to a $1.9 \sigma$ significance.

Direct evidence of CR interactions with interstellar matter comes again from Fermi-LAT, which observed a diffuse galactic $\gamma$-ray emission. The ANTARES location in the Northern latitude is ideally suited to study the expected neutrino flux from the inner galactic plane. A search has been performed searching in the regions of galactic longitude $|l|<40^{\circ}$ and latitude $|b|<3^{\circ}$ with respect to nine offzones. No excess in the on-zone region was found and the resulting limits are shown in Fig. 3. In particular, the hypothesis of a 1-1 relation between the $\gamma$-ray and neutrino flux from the Galactic Ridge is ruled out at $90 \%$ C.L., showing that ANTARES is already testing the well-established multimessenger $\gamma-v-\mathrm{CR}$ paradigm in our Galaxy. The present limit cannot rule out however models from moredetailed simulations of galactic $\mathrm{CR}$ propagation. 


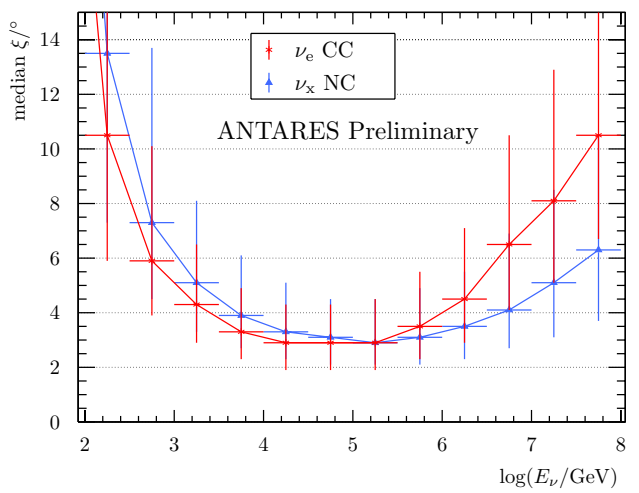

Figure 4. Angular resolutions for Monte Carlo NC (blue) and CC (red) events reconstructed with the new shower algorithm. Below $10 \mathrm{TeV}$, the resolutions worsen due to a decreasing number of photons being detected, while above $300 \mathrm{TeV}$, the events begin to saturate the detector. Over the 10-300 TeV range, arrival directions are reconstructed by the algorithm with a median angular error of $3^{\circ}$, and a resolution on deposited energy of 5\%, although the latter is limited by the total ANTARES systematic energy uncertainty of approximately $10 \%$. From T. Michael in [1].

\section{Reconstruction of cascade events}

Cascade events are mainly due to neutral-current (NC) interactions, and $v_{e}$ and $v_{\tau}$ charged-current (CC) interactions. With respect to $v_{\mu} \mathrm{CC}$ interactions, these events have worse angular resolution but the energy deposited in the detector is more-strongly correlated with the energy of the primary neutrino. In addition, cascades suffer from a smaller background from atmospheric events. These latter advantages allowed the diffuse cosmic neutrino flux detected by IceCube to be first observed in the cascade channel [2].

Cascade identification and reconstruction has been in development in ANTARES for several years. Recently, an algorithm with an unprecedented angular resolution, of typically $3^{\circ}-4^{\circ}$ accuracy has been released, see Fig. 4. This for the first time enables a point-source search using the cascade channel. A combined point-source search using both muon-track and cascade events has been performed using 1622 days of effective livetime from 2007 to 2013. The sample consisted of 6261 muon-track events, and 156 cascade events, with an estimated contamination of $10 \%$ mis-reconstructed atmospheric muons in each. The sensitivity on point-like sources are given in Fig. 5. No significant excess was observed.

\section{Dark matter and Exotics}

Neutrino telescopes can set limits on different WIMP dark-matter scenarios by limiting the neutrino flux expected from WIMP interactions in the Sun, Earth, Galactic Centre, and dwarf galaxies. Since the expected dark-matter density tends to be strongly peaked near the centers of these objects, and ANTARES has an excellent angular resolution, competitive limits can be set.

Limits on the spin-dependent (WIMP-proton) interaction cross section $\sigma_{\mathrm{SD}}^{p}$ from ANTARES observations of the Sun using the $\tau \bar{\tau}$ channel are given in Fig. 6 (left). Neutrino telescopes produce the strongest limits in the $M_{\text {WIMP }}>200 \mathrm{GeV}$ range, surpassing even the direct-detection experiments. Limits on the WIMP-WIMP velocity-averaged self-annihilation cross section $\sigma_{A} v$ from the Galactic Centre from [15] are reported in Fig. 6 (right). 


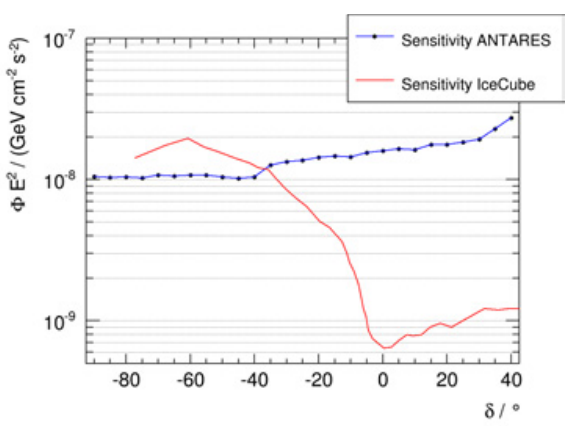

Figure 5. Sensitivity of the ANTARES targeted search to flavor-uniform neutrino point sources with $E^{-2}$ spectra in terms of flux per flavor, compared to the IceCube results from Ref. [2]. While the atmospheric background produces predominantly muon-track events, an $E^{-2}$ point source with a flavor-uniform flux would be expected to produce a cascade-to-track ratio of 3:10, significantly increasing the sensitivity of the search. This ratio is lower to one because of the long range of muons, which allows to detect $v_{\mu}$ interacting far away the instrumented volume, and the different response of the detector for tracks and cascades. The achieved sensitivity is approximately $10^{-8} \mathrm{GeV}^{-1} \mathrm{~cm}^{-2} \mathrm{~s}^{-1}$ for $\delta<-40^{\circ}$. From T. Michael in [1].
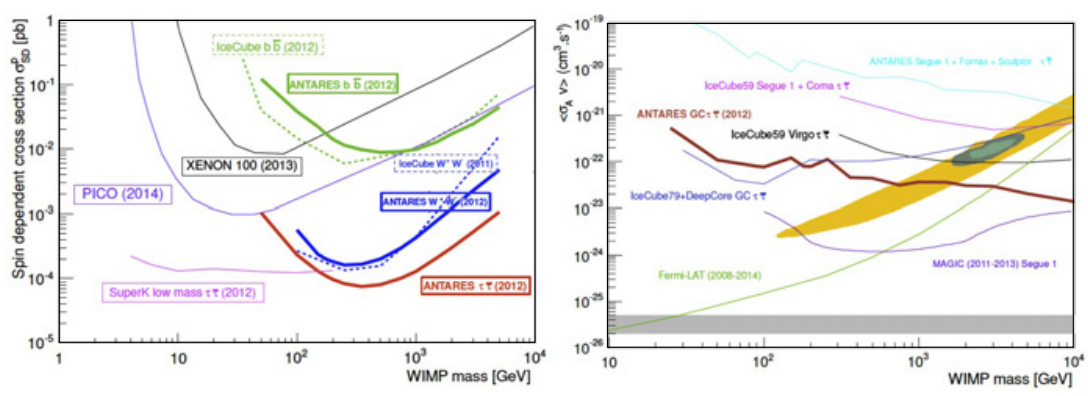

Figure 6. ANTARES limits $\sigma_{\mathrm{SD}}^{p}$ from the Sun (left) and on $\left\langle\sigma_{A} v\right\rangle$ from the Galactic Centre (right) as a function of the WIMP mass [15]. From C. Tönnis in [1].

\section{Conclusion}

The scientific framework of neutrino astrophysics has significantly changed after the IceCube detection of PeV neutrinos. The ANTARES neutrino telescope has proved to be a highly successful instrument for performing a wide range of physics analyses. The present focus is to help to understand the origin (galactic, extragalactic, mixed?) of the cosmic neutrino signal. In particular, ANTARES has an excellent angular resolution on both muon-track and cascade events, facilitated by the optical properties of deepsea water. This has allowed constraining a wide range of source scenarios, from galactic plane emission to blazars, point-like or extended objects in the Southern sky.

A new era in neutrino astronomy will begin in 2017, with the decommissioning of ANTARES, and the completion of KM3NeT Phase 1, with a unique design of multi-PMT optical modules. Phase-1 will have an instrumented volume a factor of $\sim 3$ larger than that of ANTARES [16].

\section{References}

[1] The ANTARES Collaboration: Contributions to ICRC 2015, The Hague. arXiv:1510.04508

[2] M.G. Aartsen et al., ApJ 809, 98 (2015) 
[3] S. Adrián-Martínez, et al., ApJ 786 (2014) L5

[4] S. Adrián-Martínez, et al. (ANTARES and IceCube Collaborations), arXiv: 1511.02149

[5] M. Spurio, Phys. Rev. D 90, 103004 (2014)

[6] M.C. Gonzalez-Garcia, F. Halzen, V. Niro. Astropart.Phys. 57-58 (2014) 39-48

[7] M.G. Aartsen, et al., Phys. Rev. Lett. 113, 101101 (2014)

[8] F. Krauß et al., A\&A 566, L7 (2014)

[9] S. Adrián-Martínez et al., A\&A 576, L8 (2015)

[10] S. Adrián-Martínez et al., arXiv:1506.07354 (2015) (Accepted by JCAP)

[11] S. Adrián-Martínez et al., arXiv: 1508.01180 (2015) (Accepted by JCAP)

[12] GCN circular \# 18231 and an Atel telegram \# 7987. Paper in preparation

[13] S. Adrián-Martínez et al., European Physical Journal C 74, 2701 (2014)

[14] D. Gaggero et al., arXiv: 1504.00227 (2015)

[15] S. Adrián-Martínez et al., JCAP10 (2015) 068

[16] R. Coniglione, on the behalf of KM3NeT. These proceedings 